\title{
Vessel Wall Segmentation Using Implicit Models and Total Curvature Penalizers
}

\author{
Rodrigo Moreno, Chunliang Wang, and Örjan Smedby \\ Center for Medical Imaging Science and Visualization (CMIV), \\ Department of Medical and Health Sciences (IMH) \\ Linköping University, Campus US \\ 58185, Linköping, Sweden \\ \{rodrigo.moreno, chunliang. wang, orjan.smedby\}@liu.se
}

\begin{abstract}
This paper proposes an automatic segmentation method of vessel walls that combines an implicit $3 \mathrm{D}$ model of the vessels and a total curvature penalizer in a level set evolution scheme. First, the lumen is segmented by alternating a model-guided level set evolution and a recalculation of the model itself. Second, the level set of the lumen is evolved with a term that aims at penalizing the total curvature and with a prior that forces the outer layer of the vessel towards the outside of the lumen. The model term is deactivated during this step. Finally, in a third step, the model term is reactivated in order to impose a smooth change of the radius along the vessel. Once the two segmentations have been computed, stenoses are detected and quantified at the thickest locations of the segmented vessel wall. Preliminary results show that the proposed method compares favorably with respect to the state-of-the-art both for synthetic and real CTA datasets.
\end{abstract}

\section{Introduction}

Computed Tomography Angiography (CTA) and Magnetic Resonance Angiography (MRA) have become common imaging modalities for diagnosing different cardiovascular diseases, especially those related to the presence of atherosclerotic plaques in blood vessels. Medical doctors usually evaluate the severity of a stenosis caused by an atherosclerotic plaque in a vessel by comparing the diameter of its lumen measured at narrowings and at close regions where the vessel appears healthy. Automatic and/or semi-automatic tools are required for performing this task, since manual quantification of stenoses can be extremely time consuming. This fact has fostered the research in the field in the last years.

A general approach to automatically detect and quantify stenoses is to analyze the shape of the segmented vessel wall, which is the region comprised between the lumen and the outer layer of the vessel. This analysis is usually performed through statistics on the thickness of the vessel wall at its broadest locations [1]. Thus, it is evident that accuracy on the estimation of stenoses largely depends on the quality of the vessel wall segmentation. 
By and large, the vessel wall can be obtained as the difference between the segmentation of the lumen and the outer layer of the vessel. On the one hand, state-of-the-art methods for lumen segmentation have been able to yield very accurate results, sometimes performing better than human annotators (e.g. 2, 3]). Unfortunately, the segmentation of the outer layer of the vessel is more challenging, since calcifications and soft plaques can share similar characteristics with surrounding tissue. Thus, at a large extent, the poor performance of most algorithms of the state-of-the-art in stenoses detection and quantification 1] can be attributed to poor segmentations of the outer layer of the vessels. An additional issue to be tackled for images acquired from the coronary arteries is the low resolution. For example, the diameter of the vessels can range between ten and less than one voxel at the beginning and end of the vessel respectively. This makes sub-voxel accuracy methods, such as level sets, necessary for solving this problem.

In this line, this paper aims at proposing a level set-based segmentation method for extracting the outer layer of the vessel, which, in conjunction with a previously proposed lumen segmentation method [2], is used to extract and analyze the vessel wall. The segmented lumen is evolved into the outer layer through a level set that combines a curvature regularization term, an implicit vessel model and a threshold-based image term. In addition, the lumen is used as a prior in order to maintain the outer layer outside the lumen. The paper is organized as follows. Section 2 introduces the proposed method. Section 3 presents some results of the proposed method both with synthetic and CTA data. Finally, Section 4 discusses the results and makes some final remarks.

\section{Method}

As already mentioned, the proposed method uses level sets. In level set segmentation methods, contours are represented by the zero level of a level set function $\phi$. This function can be, for example, a signed distance function from the contour of interest. The method aims at iteratively modifying this function in order to make the zero level converge to the boundary of the object of interest. The following subsections describe the segmentation of the lumen and the outer layer of the vessel.

\subsection{Model-Guided Lumen Segmentation}

This subsection briefly summarizes the method proposed in [2] which is used here for segmenting the lumen only. The algorithm starts with an input image and initial centerlines for every coronary artery branch. From the initial centerlines, an implicit vessel model is generated, which is used to guide the level set propagation. After a number of iterations of the level set evolution, the centerlines and the radius of the vessels are recalculated in order to generate an improved vessel model. These two steps are alternated repeatedly until convergence. 
As shown during the "3D Cardiovascular Imaging: a MICCAI segmentation challenge workshop" 1 , this approach yields state-of-the-art results and in some cases it is even able to outperform human annotators.

The method uses the following level set evolution equation:

$$
\frac{\partial \phi}{\partial t}=\left(\alpha v_{\text {image }}+\beta v_{\text {smoothness }}+\gamma v_{\text {prior }}\right)|\nabla \phi(x)|,
$$

where $\phi$ is the level set function, $\alpha, \beta$ and $\gamma$ are weighting parameters and the terms $v_{\text {image }}, v_{\text {smoothness }}$ and $v_{\text {prior }}$ are defined as follows.

First, $v_{\text {image }}$ is a threshold-based image term which tries to keep the gray-scale values inside the lumen in a certain range. Such a range is automatically estimated through some statistics on the gray-scale values at the centerline. Second, $v_{\text {smoothness }}$ is a curvature-based smoothness term. Since the difference between the principal curvatures in healthy vessels is large, minimum curvature was used instead of the commonly used mean curvature in order to avoid potential shrinkages along the vessels related to the natural large curvatures present across the vessels. This approach is in the same line as the one proposed in [4]. Finally, $v_{\text {prior }}$ is a term to penalize deviations of the segmentation from a model of the vessel. The model is a generalized cylinder implemented as an implicit function (i.e. a level set) generated from a centerline and a radius function. As aforementioned, both centerline and radius are also evolving with the segmentation. The model acts not only as a prior for the level set evolution but it also guarantees the preservation of the topology of the vessel by preventing cuts at the zero level set. Notice that the input centerlines are not required to be perfectly centered. Actually, most of the methods proposed in the bibliography (cf. [5] for a review of the most representative approaches for this task) yield results of enough quality to be used in the method.

\subsection{Smoothness Term for Outer Layer Segmentation}

The most important difference between the method proposed in [2] for segmenting the outer layer and the one proposed in this paper is the applied smoothness term. Thus, the aim of this subsection is to motivate the necessity of using different smoothness terms for lumen and outer layer segmentation.

Indeed, the desired smoothing effect for the outer layer segmentation is essentially different from the one applied for the lumen segmentation. By applying the Euler-Lagrange equation, it can be proven that the solution of the mean curvature motion (MCM) PDE equation:

$$
\frac{\partial \phi}{\partial t}=H|\nabla \phi(x)|
$$

is a minimizer of the energy functional $E_{A}=\int_{S} d S$, where $H$ is the mean curvature and $S$ is the surface [6]. That is, the MCM PDE equation can be used to minimize the area of the surface $S$. Using the minimum curvature instead of

\footnotetext{
${ }^{1}$ The website of the challenge is: http://coronary.bigr.nl/stenoses/index.php
} 
$H$, as performed in [2] can be regarded as an anisotropic variant of (2) which also aims at minimizing the surface area of $S$, but in this case anisotropically.

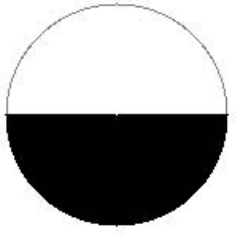

(a)

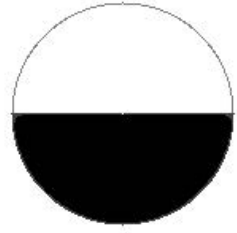

(b)

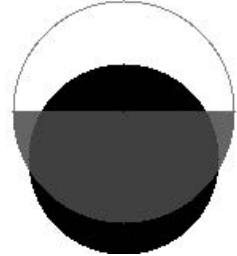

(c)

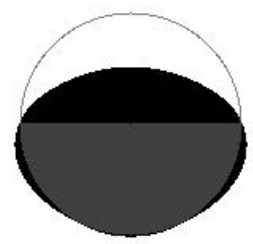

(d)

Fig. 1. (a): A cross section of a simulated stenosis. The black area corresponds to the lumen of the vessel. (b): Result after applying 50 iterations of a level set with a threshold-based image term and MCM. (c): Result after applying 50 iterations of a level set with the same terms plus the model term. (d) Result after applying 50 iterations with the total curvature smoothness term and disabling the model term. The lumen is shown in gray on Figures (b-d).

Although this smoothness term has interesting properties and is appropriate for lumen segmentation, it does not have some desirable properties required for segmenting the outer layer of the vessel. Let us illustrate this with a $2 \mathrm{D}$ example. Figure1(a) shows a cross section of a synthetic example where the plaque covers the top part of the vessel. Assume in this case that the gray-scale values of the plaque are similar to those from the surrounding, so the image term is unable to move the level set towards the outer layer of the vessel. The result is that, despite of the big size of the plaque, the lumen and outer layer segmentation yield similar results when the same smoothness term is used as shown in Figure 1(b). Unfortunately, in this case, the model term used in (1) for segmenting the lumen cannot help either to get better segmentations of the outer layer. Even if accurate estimations of the radius of the vessel are available, the centerline will remain stuck at the center of the segmented lumen instead of moving towards the center of the vessel. This can lead to the wrong segmentation shown in Figure1(c). Our approach to solve this problem is to use a more appropriate smoothness term. The main objective of the new smoothness term is to make centerlines leave the local minima before the evolution of the level set can continue. This effect can be obtained through the intrinsic Laplacian of mean curvature motion (ILMCM), as shown in Figure 1(d).

Consider the total curvature energy functional [7]:

$$
E_{t c}=\frac{1}{2} \int_{S}\left(\kappa_{1}^{2}+\kappa_{2}^{2}\right) d S
$$

where $\kappa_{1}$ and $\kappa_{2}$ correspond to the maximum and minimum principal curvatures at $S$ respectively. The first variation of this functional corresponds to the ILMCM, which is given by the following fourth-order PDE [8] [9]: 


$$
\frac{\partial \phi}{\partial t}=\Delta H|\nabla \phi(x)|
$$

where $\Delta H$ is the Laplacian of the mean curvature.

An interesting property of the ILMCM is that, while it is well-known that level sets vanish under standard MCM after a large enough number of iterations, they converge to spheres under ILMCM which size depend on the initial level set [7]. In the proposed method, ILMCM is combined with appropriate prior terms which make the level set converge to a generalized cylinder where the total curvature is minimized.

Although there are alternatives to the ILMCM, it is the most suitable approach for vessel segmentation. For example, one alternative, is to use the energy functional:

$$
E_{p}=\int_{S}\left(\kappa_{1}-\hat{\kappa_{1}}\right)^{2} d S,
$$

with $\hat{\kappa_{1}}=1 / \hat{r}$, and $\hat{r}$ being the expected (or estimated) radius of the vessel. Unfortunately, efficient numerical schemes used in level sets for estimating curvature are not accurate, especially in objects with very high $\kappa_{1}$, something that is common in vessels. This makes difficult to use this approach for vessel segmentation. Another alternative is the Willmore flow, which penalizes the squared of the mean curvature instead of the total curvature [10 12] or its generalization using higher powers of $H[13]$. Specifically, the Willmore energy is given by:

$$
E_{w}=\frac{1}{2} \int_{S} H^{2} d S=\frac{1}{8} E_{t c}+\frac{1}{4} \int_{S} K d S .
$$

where $K$ is the Gaussian curvature and $E_{t c}$ is given by (3). From this equation, it can be seen that ILMCM is more appropriate for vessel segmentation, since the extra penalizer on the right of (6), which considers the interaction between the two principal curvatures, is not relevant in this application and it imposes an unnecessary computational burden. A final alternative comes from the Euler's elastica theory, which integrates ILMCM and MCM in the same framework [14, 15]. Its energy functional in $2 \mathrm{D}$ is given by:

$$
E_{e}=\int_{S}\left(a+b \kappa^{2}\right) d S
$$

with $a$ and $b$ being parameters. The proposed method can be seen as a particular case in which $a=0$ for segmenting the lumen and $b=0$ for segmenting the outer layer of the vessel.

\subsection{Segmentation of the Outer Layer}

For segmenting the outer layer, the level set is initialized with the final level set obtained during the lumen segmentation. Although, the general equation (1) is also applied for the outer layer segmentation, each term is adapted for this specific task as described below. 
Design of the Image Term. Instead of using a range of values, only a lower threshold is considered in the image term. This allows us to include calcified plaques in the segmentation. That is, $v_{\text {image }}=I(x)-T_{\text {inf }}$, where $T_{\text {inf }}$ is a constant that been set to $150 \mathrm{HU}$ for CTA datasets used in Section 3 .

Design of the Smoothness Term. As already mentioned, the ILMCM smoothness term is used instead of the anisotropic MCM used for lumen segmentation in order to make the centerlines leave local minima.

Despite its advantages, one important problem of ILMCM is that basic numerical schemes tend to be unstable. Also, since the PDE requires fourth-order derivatives, the estimation of curvature yields inaccurate values in small objects, which is the case at end of coronary arteries. In recent years, more stable and accurate schemes have been proposed for solving (44). For example, [16] proposed to split ILMCM into two alternated second order PDEs that tend to converge to the same solution of ILMCM. This numerical scheme was used in the experiments of Section 3. In any case, a small time step must be used for the sake of stability. It also is important to remark that, for vessel segmentation, only the curvature of the cross sections (which corresponds to the maximum principal curvature) requires regularization. That means that the energy functional $E_{m c}=\int_{S} \kappa_{1}^{2} d S$ can be used instead of the total curvature energy functional. However, since $\kappa_{1} \gg \kappa_{2}$ in vessels (especially at their endings), both functionals are approximately equivalent. An important additional advantage of including a penalizer for the minimum curvature $\kappa_{2}$ is that the numerical schemes become more stable.

Design of the Prior Term. The ILMCM described in the previous subsection must be guided with an appropriate prior term. One of the drawbacks of our method in [2] is that the outer layer and the lumen are computed independently and in some cases this results in larger diameters in the segmented lumen than in the outer layer. Considering that this paradox is mainly caused by numerical errors, the outer layer was corrected by a statistical analysis on the differences of diameters. The proposed method includes an extra prior term that makes unnecessary this correction. The prior term is defined as:

$$
v_{\text {prior }}=p_{1} v_{\text {model }}+p_{2} v_{\text {lumen }}
$$

where $p_{1}$ and $p_{2}$ are parameters, $v_{\text {model }}$ is the same term used in the lumen segmentation and $v_{\text {lumen }}$ penalizes the intrusions of the outer layer inside the segmented lumen:

$$
v_{\text {lumen }}(x)=\left\{\begin{array}{ll}
1 & \text { if } \phi(x) \leq 0 \text { and } \phi_{0}(x) \leq 0 \\
0 & \text { otherwise }
\end{array},\right.
$$

where $\phi_{0}$ is the level set of the lumen segmentation. That is, the level set is pushed outwards if both level sets intersect and no force is exerted to attract the level set of the outer layer towards the boundary of the lumen. In that sense, this 
term can be seen as an asymmetric threshold-based level set evolution function. As well as for the lumen segmentation, this prior term prevents changes in the topology of the level set.

Parameter Setting. The segmentation of the outer layer of the vessel is performed in two steps. In the first one, parameters are set in such a way that the ILMCM smoothness term and the prior $v_{\text {lumen }}$ are dominant, the prior $v_{\text {model }}$ is deactivated and the influence of the image term is reduced but not deactivated. This means that $\alpha$ is low, $\beta$ and $\gamma \times p_{2}$ are high, while $p_{1}$ is set to zero. The objective of this step is to help the centerlines to escape from possible local minima. In a second step, the model and image terms are reactivated for a few iterations in order to get a better estimation of the outer layer. In this case $\alpha, \beta$ and $\gamma \times p_{1}$ are set with medium values and $\gamma \times p_{2}$ with high values in order to push the level set outwards the lumen. Notice that the importance of a specific parameter is measured with respect to the sum of all parameters.

\section{Experimental Results}

Figure 2 shows a simulated severe stenosis and the output of the proposed method after 300 iterations without the model term. Since the centerline is now close to the center of the vessel, the model term can be switched on for a few iterations (three in this experiment) in order to impose a smooth change in the radius function along the vessel. The estimated narrowing was (86\%), which lies close to the ground truth $(80 \%)$.

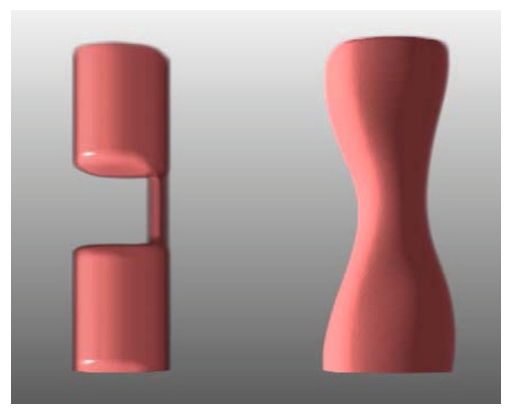

Fig. 2. Left: simulated severe stenosis ( $80 \%$ of narrowing). Right: result of the proposed method after 300 iterations.

In addition, the method was applied to the 18 coronary artery CTA training datasets provided by the organizers of the 3D Cardiovascular Imaging: A MICCAI Segmentation Challenge workshop, at the MICCAI 2012. The initial centerlines were provided by the team Rcadia [17]. Figure 3] shows the lumen and outer layer segmentation of the dataset number 10. 

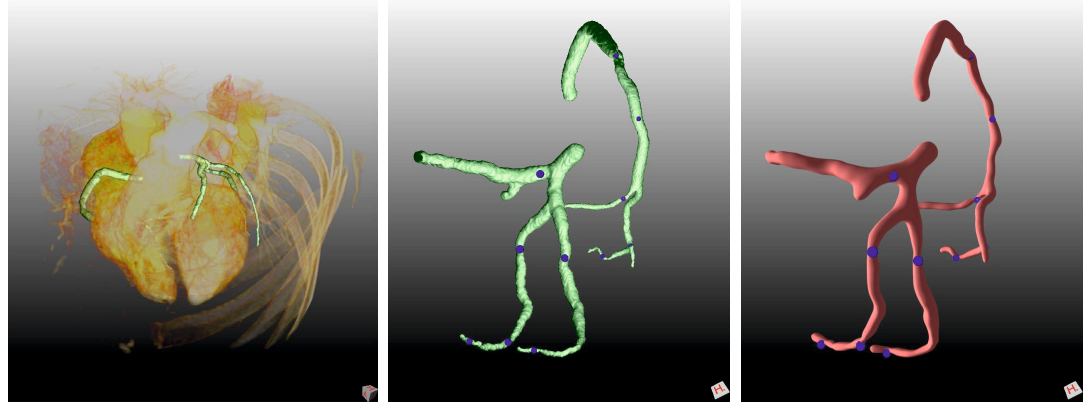

Fig. 3. Left: rendering of a CTA dataset with the segmented lumen. Center: segmented lumen. Right: segmented outer layer of the vessels. A different pose has been used in the last two renderings for a better visualization of the stenoses (marked in blue).

Table 1. Summary of stenoses detection results. The maximum value of each column is marked in bold.

\begin{tabular}{l|c|c|c|c}
\hline Method & QCA Sensitivity & QCA PPV. & CTA Sensitivity & CTA PPV \\
\hline Observer1 & 0.56 & 0.42 & $\mathbf{0 . 7 2}$ & 0.50 \\
Observer2 & 0.58 & $\mathbf{0 . 4 9}$ & 0.64 & $\mathbf{0 . 6 4}$ \\
Observer3 & 0.43 & 0.46 & 0.46 & 0.51 \\
Cetin et al. 2012 & 0.54 & 0.43 & 0.70 & 0.54 \\
Mohr et al. 2012 & 0.58 & 0.24 & 0.47 & 0.19 \\
Duval et al. 2012 & 0.52 & 0.17 & 0.63 & 0.15 \\
Wang et al. 2012 & 0.36 & 0.31 & 0.28 & 0.23 \\
Proposed method & $\mathbf{0 . 8 0}$ & 0.20 & 0.50 & 0.08 \\
\hline
\end{tabular}

The method has been compared with the results from the challenge using the methodology described in [1], that is through sensitivity and positive predictive values (PPV) of quantitative coronary angiography (QCA) and computed tomography coronary angiography (CTA) analyses with respect to human annotated ground-truths. Stenoses have been reported at the thickest points of the segmented vessel wall. Table 1 shows the results of the proposed method compared to the four methods with better ranking reported on the website of the challenge 2 . As shown in the table, the sensitivity is largely improved compared to our previous method. It is interesting to see that the new method outperforms human observers sensitivity in some cases. On the other hand, the decay in the PPV values indicates an increase in the amount of false positives. Our current research aims at reducing this amount while keeping higher values of sensitivity.

\section{Discussion}

A new segmentation method for the outer layer vessel level set has been proposed. It is used in conjunction with a previously proposed method for lumen

\footnotetext{
${ }^{2}$ These results were taken from this website the 1st of February 2013.
} 
segmentation to automatically detect and quantify stenoses in coronary CTA datasets. By introducing a higher-order smoothness term, the centerlines are forced to move towards the center of the vessel instead of at the center of the lumen. Preliminary results are promising, especially regarding sensitivity.

It is important to remark that, since only a single point per lesion is considered, which is marked at the local maximum of the segmented vessel wall, this point might not coincide with those marked by the observers, especially when the plaque is relatively long. Actually this can have a large impact in the statistics reported in Table 1 .

Several aspects need to be improved for using the proposed method in clinical practice. One drawback of the method is that its performance directly relies on the quality of the input centerlines. Unfortunately, the centerlines provided by the team Rcadia have problems in case of severe stenoses and occlusions. Another issue of the current implementation is that the centerlines are not allowed to extend or shrink. Although points in the middle of the centerline are updated iteratively, starting and ending points of centerlines are kept fixed as the original input. Allowing centerlines to grow in the most likely directions can be useful to discover additional vessel structures at every iteration.

Furthermore, a current drawback of the method is that estimating the most suitable thresholds is a difficult task. For human observers, dynamic window setting can be helpful to improve the accuracy of grading. A similar technique can be implemented in the proposed method by running the segmentation at multiple threshold levels, which might provide a profile of the composition of the vessel wall. Moreover, in many segments, the threshold of the level set can be lowered to as low as $0 \mathrm{HU}$ without causing leaking problems. This approach could potentially be useful for finding the outer border of the vessel wall where adipose tissue $(-70$ to $-30 \mathrm{HU})$ is present. Finally, the current implementation can be further speeded up by using smaller regions of interest, better estimation of the model, parallel computing and by using local convergence detection through coherent propagation [18].

Acknowledgements. This research has been supported by the Swedish Research Council (VR), grant no. 2011-5197 and the Swedish Heart-Lung Foundation (HLF), grant no. 2011-0376.

\section{References}

1. Kirisli, H., Schaap, M., Metz, C., Dharampal, A., Meijboom, W., Papadopoulou, S., Dedic, A., Nieman, K., de Graaf, M., Meijs, M., Cramer, M., Broersen, A., Cetin, S., Eslami, A., Florez-Valencia, L., Lor, K., Matuszewski, B., Melki, I., Mohr, B., Oksuz, I., Shahzad, R., Wang, C., Kitslaar, P., Unal, G., Katouzian, A., Orkisz, M., Chen, C., Precioso, F., Najman, L., Masood, S., Unay, D., van Vliet, L., Moreno, R., Goldenberg, R., Vucini, E., Krestin, G., Niessen, W., van Walsum, T.: Standardized evaluation framework for evaluating coronary artery stenosis detection, stenosis quantification and lumen segmentation algorithms in computed tomography angiography. Medical Image Analysis (2012) (submitted) 
2. Wang, C., Moreno, R., Smedby, Ö.: Vessel segmentation using implicit modelguided level sets. In: Proceedings of 3D Cardiovascular Imaging: a MICCAI Segmentation Challenge Workshop (2012)

3. Mohr, B., Masood, S., Plakas, C.: Accurate lumen segmentation and stenosis detection and quantification in coronary CTA. In: Proceedings of 3D Cardiovascular Imaging: a MICCAI Segmentation Challenge Workshop (2012)

4. Lorigo, L.M., Faugeras, O.D., Grimson, W.E., Keriven, R., Kikinis, R., Nabavi, A., Westin, C.F.: CURVES: curve evolution for vessel segmentation. Medical Image Analysis 5(3), 195-206 (2001)

5. Schaap, M., Metz, C.T., van Walsum, T., van der Giessen, A.G., Weustink, A.C., Mollet, N.R., Bauer, C., Bogunović, H., Castro, C., Deng, X., Dikici, E., O’Donnell, T., Frenay, M., Friman, O., Hoyos, M.H., Kitslaar, P.H., Krissian, K., Kühnel, C., Luengo-Oroz, M.A., Orkisz, M., Smedby, Ö., Styner, M., Szymczak, A., Tek, H., Wang, C., Warfield, S.K., Zambal, S., Zhang, Y., Krestin, G.P., Niessen, W.J.: Standardized evaluation methodology and reference database for evaluating coronary artery centerline extraction algorithms. Medical Image Analysis 13(5), 701-714 (2009)

6. Caselles, V., Kimmel, R., Sapiro, G., Sbert, C.: Minimal surfaces based object segmentation. IEEE Transactions on Pattern Analysis and Machine Intelligence 19(4), 394-398 (1997)

7. Polden, A.: Curves and surfaces of least total curvature and fourth-order flows. PhD thesis, Tübingen University (1996)

8. Coleman, B., Falk, R., Moakher, M.: Spacetime finite element methods for surface diffusion with applications to the theory of the stability of cylinders. SIAM Journal on Scientific Computing 17(6), 1434-1448 (1996)

9. Chopp, D.L., Sethian, J.A.: Motion by intrinsic Laplacian of curvature. Interfaces and Free Boundaries 1, 1-18 (1999)

10. Droske, M., Rumpf, M.: A level set formulation for Willmore flow. Interfaces and Free Boundaries 6, 361-378 (2004)

11. Droske, M., Bertozzi, A.: Higher-order feature-preserving geometric regularization. SIAM Journal on Imaging Sciences 3(1), 21-51 (2010)

12. Burger, M., Stöcker, C., Voigt, A.: Finite element-based level set methods for higher order flows. Journal of Scientific Computing 35(2-3), 77-98 (2008)

13. Caselles, V., Haro, G., Sapiro, G., Verdera, J.: On geometric variational models for inpainting surface holes. Computer Vision and Image Understanding 111(3), 351-373 (2008)

14. Chan, T.F., Kang, S.H., Shen, J.: Euler's elastica and curvature based inpaintings. SIAM Journal on Applied Mathematics 63, 564-592 (2002)

15. El-Zehiry, N.Y., Grady, L.: Vessel segmentation using 3D elastica regularization. In: Proceedings of International Symposium on Biomedical Imaging (ISBI), pp. 1288-1291 (2012)

16. Tasdizen, T., Whitaker, R.T., Burchard, P., Osher, S.: Geometric surface smoothing via anisotropic diffusion of normals. In: Proceedings of IEEE Visualization, pp. $125-132(2002)$

17. Goldenberg, R., Eilot, D., Begelman, G., Walach, E., Ben-Ishai, E., Peled, N.: Computer-aided simple triage (CAST) for coronary CT angiography (CCTA). International Journal Computer Assisted Radiology and Surgery, 1-9 (2012)

18. Wang, C., Frimmel, H., Smedby, Ö.: Level-set based vessel segmentation accelerated with periodic monotonic speed function. Proceedings of SPIE-Medical Imaging: Image Processing, vol. 7962, pp. 79621M-1-79621M-7 (2011) 